\title{
The Direction of Spontaneous Magnetisation of Lanthanide lons at a Site of Cubic Symmetry
}

\author{
C. Carboni*, E. Al-Abry and S. Arafin
}

Department of Physics, College of Science, Sultan Qaboos University, P.O.Box 36, Al Khod 123, Muscat, Sultanate of Oman, *Email: carlo@squ.edu.om.

\section{التجاdمغنلليسية مواد الأنشاليس}

\section{كارلوكارسوف ، إيمل العبري وعسيد العارفين}

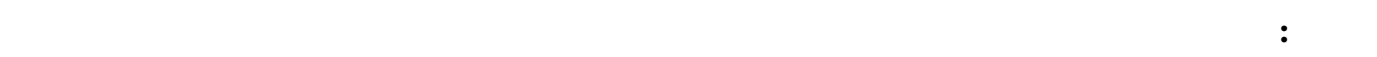

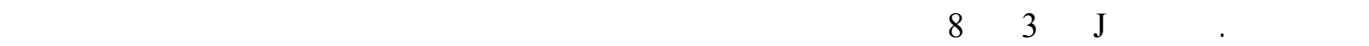

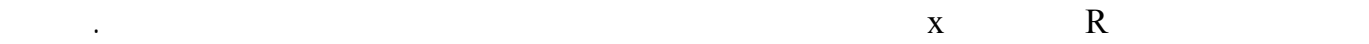

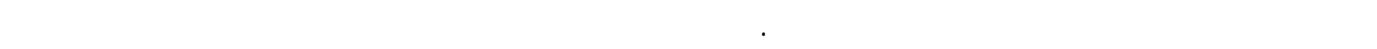

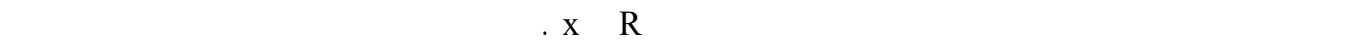

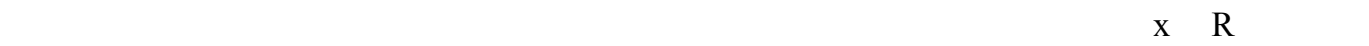

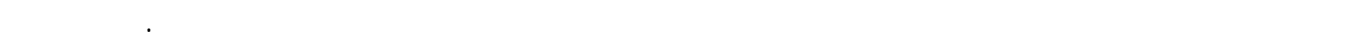
الأخيرة تكون مطقة قريبة من الحد يكون فيها التجه المغنطيسية غير مسنقر.

ABSTRACT: The zero temperature direction of spontaneous magnetisation of lanthanide ions at a site of cubic symmetry is investigated as a function of the electrostatic and magnetic interactions. For all the values of $\mathrm{J}$ between $\mathrm{J}=3$ and $\mathrm{J}=8$, two-dimensional diagrams giving the orientation of spontaneous magnetisation are obtained as a function of the parameters $\mathrm{R}$ representing the relative strength of the magnetic to electrostatic interaction and the parameter $\mathrm{x}$ representing the relative strength of the fourth to the sixth order terms in the crystal field. The boundaries between regions of the parameter space with different directions of spontaneous magnetisation are investigated. It is found that at some boundaries there is a gradual rotation of the direction of spontaneous magnetisation and that at other boundaries there is a sudden change of orientation of spontaneous magnetisation at a critical value of the $(\mathrm{R}, \mathrm{x})$ parameters. Two types of behaviour are observed when there is a critical value of $(\mathrm{R}, \mathrm{x})$. There are boundaries where there is at the critical value a degenerate plane in which all the orientations can be direction of spontaneous magnetisation and some boundaries where two different principal crystallographic axes can be direction of spontaneous magnetisation at the critical value. In the latter case there is a region near the boundary where an unstable equilibrium orientation for the magnetisation can be found. 


\section{CARBONI, E. AL-ABRY and S. ARAFIN}

KEYWORDS: Spin reorientation, crystal field, $\mathrm{Y}_{\mathrm{x}} \mathrm{Ho}_{1-\mathrm{x}} \mathrm{Co}_{2}, \mathrm{Gd}_{\mathrm{x}} \mathrm{Ho}_{1-\mathrm{x}} \mathrm{Co}_{2}, \mathrm{Gd}_{\mathrm{x}} \mathrm{Y}_{\mathrm{y}} \mathrm{Ho}_{1-\mathrm{x}-}$ ${ }_{\mathrm{y}} \mathrm{Co}_{2} \mathrm{HoCo}_{2}$.

\section{Introduction}

$\mathbf{T}_{\mathrm{b}}$ he direction of spontaneous magnetisation in magnetic rare-earth compounds is the result of a delicate balance between the electrostatic and magnetic interactions experienced by the lanthanide ion. The electrostatic interaction is due to the interaction of the localised $4 \mathrm{f}$ electrons of the lanthanide ion with the electric field gradients set up by the lattice at the ion's site. The magnetic interactions can arise from dipoledipole or exchange interactions. These interactions between the ion and its surrounding in the solid are small compared to the intra-ionic interactions and can be regarded a perturbation on the free ion states (Morrisson and Leavitt, 1982). The free ion states are almost pure Russell-Saunders states; L, S and J are good quantum numbers and remain good quantum numbers in the solid. The effect of the interactions in the solid is to split the (2J+1)fold degenerate free-ion states without causing significant admixture of the sates. Because of the large spin-orbit interaction (1000K or more) only the ground manifold is significantly populated at room temperature or below therefore all the populated states have the same $\mathrm{L}, \mathrm{S}$ and $\mathrm{J}$ and the states of the ion in the solid are expressed as linear combinations of the $\mid \mathrm{M}_{\mathrm{J}}>$ states.

$$
|E\rangle=\sum_{i=-J}^{i=+J} A_{i}\left|M_{i}\right\rangle
$$

The omitted L, S and J labels have the values given by Hund's rule for the ground manifold.

The magnetic properties of a compound and in particular its direction of spontaneous magnetisation are determined by the composition of the low-lying states in the ground manifold. For each state the direction of spontaneous magnetisation is the orientation of $\langle\mathbf{J}\rangle$ for which the state has the lowest energy. Different states may have different directions of spontaneous magnetisation. At any given temperature the direction of spontaneous magnetisation of a compound is the thermal average over the populated states. When the temperature is changed, the concomitant change in the population of the states may give rise to the "spin reorientations" observed in several rare-earth compounds.

In the present paper we consider a lanthanide ion at a site of cubic symmetry in a compound at sufficiently low temperature so that only the ground state of the ground manifold the ion is significantly populated. This zero temperature assumption is justified because it is valid for most rare earth compounds at liquid helium temperatures. The evolution of the direction of spontaneous magnetisation at zero temperature will be investigated as a function of the relative strengths of the interactions in the solid. Following McCausland and Mackenzie (1980) the magnetic interactions will be treated in a rather cavalier manner and will be described by a single isotropic vector parameter. With this simplification it is possible to construct, following the work of Lea et al. (1962), a dimensionless two-parameters Hamiltonian to obtain the orientation of spontaneous magnetisation of the lanthanide ion. The direction of spontaneous magnetisation can then be presented on a simple twodimension diagram as a function of the two parameters.

\section{Theory}

The theoretical approach to write the perturbation Hamiltonian for the ion follows Lea, Leask and Wolf (1962), McCausland and Mackenzie (1980) and Bunbury et al. (1995). The ground manifold is assumed to be a pure Russell-Saunders state and the interactions with the solid are treated as a perturbation in first order. The small amount of intermediate coupling and J-mixing is ignored $[4,5]$

\subsection{The electronic hamiltonian}

The strengths of the crystal-field and the magnetic interactions of the ion in the solid are of the same order and the operators describing these two interactions do not commute. The ion in the solid is described by the combined effective electronic Hamiltonian: 


$$
\mathscr{H}_{\mathrm{el}}=\mathscr{H}_{\mathrm{cf}}+\mathscr{H}_{\mathrm{Z}}
$$

where $\mathscr{H}_{\mathrm{cf}}$, the crystal-field Hamiltonian, stands for the electrostatic interaction and $\mathscr{H}_{\mathrm{z}}$ stands for all the magnetic interactions. Following Lea et al. (1962), the crystal field Hamiltonian at the site of cubic symmetry is written:

$$
\mathscr{H}_{\mathrm{cf}}(W, x)=\frac{W x}{F_{4}}\left(\mathbf{O}_{4}^{0}+5 \mathbf{O}_{4}^{4}\right)+\frac{W}{F_{6}}(1-|x|)\left(\mathbf{O}_{6}^{0}-21 \mathbf{O}_{6}^{4}\right)
$$

Where the $\mathbf{O}_{n}^{m}$ are Stevens operator equivalents [6]. W and $\mathrm{x}$ are the phenomenological crystal field parameters; the parameter $\mathrm{W}$ gives the strength of the crystal field and the dimensionless parameter $\mathrm{x}$ gives the relative strength of the fourth-order to the sixth-order term. $\mathrm{F}_{4}$ and $\mathrm{F}_{6}$ are numerical constants tabulated by Lea et al. (1962) . For completeness the operator equivalents and the values of the coefficients $F_{4}$ and $F_{6}$ for the relevant values of $\mathrm{J}$ are given in appendix 1 .

As already mentioned all the magnetic interactions are considered to be isotropic and therefore, following McCausland and Mackenzie (1980) can be represented by a vector $\alpha$. The magnetic interactions are thus described by the Hamiltonian.

$$
\not \mathscr{C}_{\mathrm{z}}=\alpha \cdot \mathbf{J}
$$

It is enphasised that the assumptions made when writing equation 4 are not necessarily valid for all real compounds. In particular in the presence of anisotropy in the exchange interaction or in magneto-electric materials one will have to introduce extra terms in the magnetic interaction to describe the anisotropy or the coupling to the electrostatic terms. Such refinements will inevitably introduce extra parameters in the Hamiltonian and therefore make the outcome of this investigation rather complex and difficult to represent on simple two-dimension diagrams. However, one can reasonably expect that apart from some possible extreme exceptions the very much simplified expression of the magnetic interactions used here will give a description that is not too far from the reality. Therefore, for the general case of well-behaved materials the interactions of the 4-f shell are described by the Hamiltonian

$$
\mathscr{H}_{\mathrm{el}}(W, x)=\frac{W x}{F_{4}}\left(\mathbf{O}_{4}^{0}+5 \mathbf{O}_{4}^{4}\right)+\frac{W}{F_{6}}(1-|x|)\left(\mathbf{O}_{6}^{0}-21 \mathbf{O}_{6}^{4}\right)+\boldsymbol{\alpha} \cdot \mathbf{J}
$$

Since we are concerned with the direction of spontaneous magnetisation as a function of the relative strengths of the crystal-field and magnetic interactions it is preferable to consider the dimensionless Hamiltonian

$$
\mathscr{H}_{e l}=\mathbf{R} \cdot \mathbf{J}+\left[\frac{x}{F_{4}}\left(\mathbf{O}_{4}^{0}+5 \mathbf{O}_{4}^{4}\right)+\frac{1}{F_{6}}(1-|x|)\left(\mathbf{O}_{6}^{0}-21 \mathbf{O}_{6}^{4}\right)\right]
$$

where the parameter $\mathbf{R}$ is a vector with the same direction as $\alpha$ and magnitude $(|\alpha| / \mathrm{W})$. $|\mathbf{R}|$ is the relative strength of the magnetic to the electrostatic interaction. There are only two parameters, $|\mathbf{R}|$ and $\mathrm{x}$, in the Hamiltonian. The direction of spontaneous magnetisation can therefore be readily mapped on a two-dimensional diagram as a function of $|\mathbf{R}|$ and $\mathrm{x}$.

\subsection{The direction of spontaneous magnetisation}

To determine the direction of spontaneous magnetisation for a given value of $|\mathbf{R}|$ and $\mathrm{x}$, one has to find the orientation of $\mathbf{R}$ that yields the lowest energy for the ground state of the electronic Hamiltonian subjected to the condition that the computed orientation of $\mathbf{J}$ is parallel to the input orientation of $\mathbf{R}$. The later condition is stating that the spontaneous magnetisation is parallel to the local field at the ion's site. We note in passing that $\mathbf{J}$ will be parallel to $\mathbf{R}$ in the case of pure compounds. In the case of an impurity ion in a host with competing magnetic anisotropy $\mathbf{J}$ is not necessarily parallel to $\mathbf{R}$; such cases are not considered in the present work. In the remainder of this paper, the orientations of $\mathbf{R}$ where $\mathbf{J}$ is found to be parallel to $\mathbf{R}$ will be referred to as self-consistent orientations. By symmetry, the principal crystallographic orientations, $<110>,<100>$ and $<111>$ are selfconsistent orientations. However, these orientations are not necessarily the only ones or do any of them correspond necessarily to the lowest energy. 

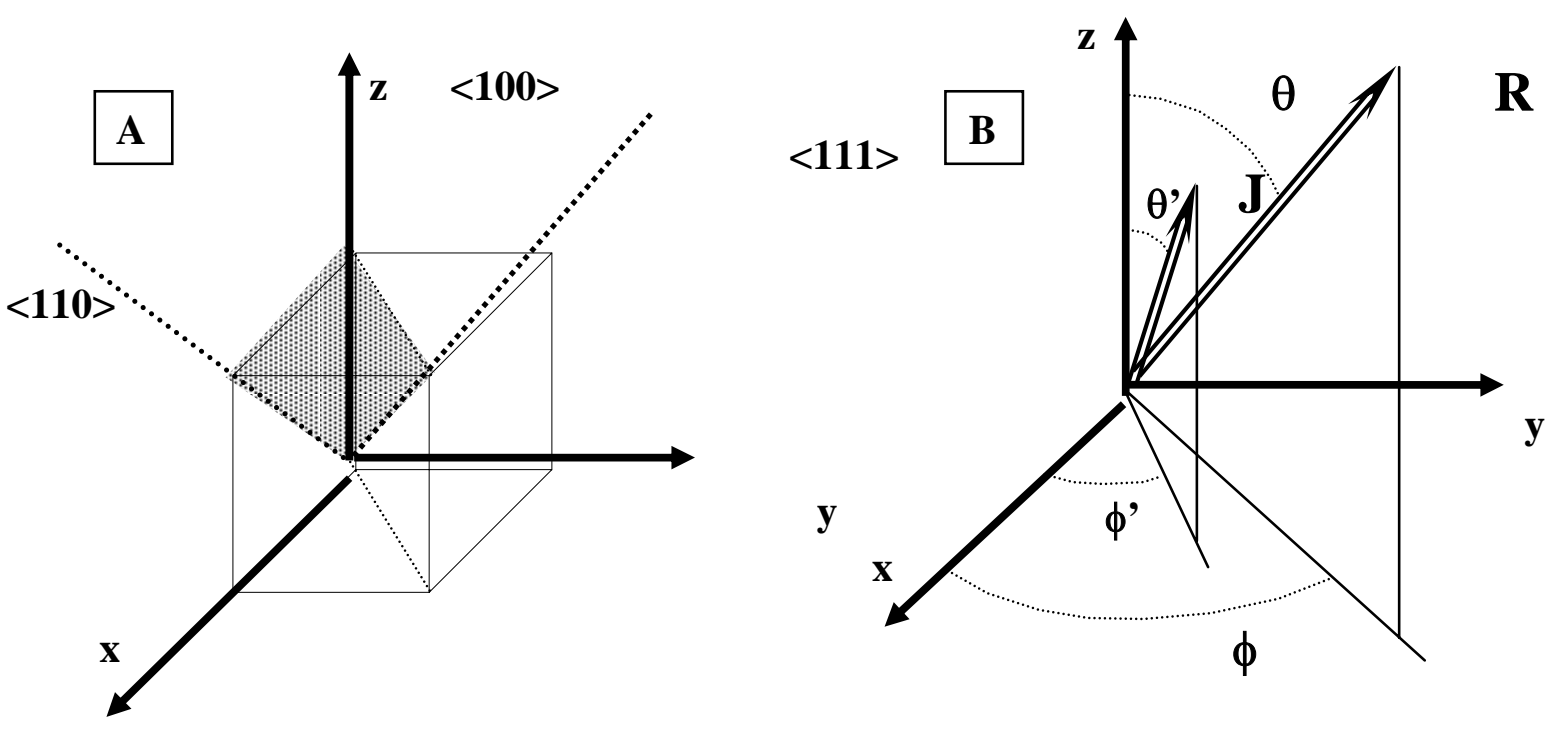

Figure 1. The convention and notation used for the computation. A: The $\mathrm{x}, \mathrm{y}$ and $\mathrm{z}$ axis are taken along the edges of the cubic unit cell. By symmetry only the directions within the hashed tetrahedron need to be considered. B: The polar angles of the input vector $\mathrm{R}$ are called $\theta$ and $\phi$ and the computed polar angles of $\mathrm{J}$ are labelled $\theta^{\prime}$ and $\phi$.

\section{Computation strategy}

A pedestrian approach to mapping the magnetic orientation phase diagram as a function of $|R|$ and $x$ would be to input systematically values of $\mathrm{x}$ and $|\mathrm{R}|$ and to let the software find which one of the self-consistent orientations has the lowest energy for each $(\mathrm{R}, \mathrm{x})$. However, a rough estimate of the computer time required for this approach is prohibitively too long. A more computer-time efficient approach had to be used for the present work. In this paragraph we give a description of the approach taken for the computation and define the notation used.

The reference frame to write the Hamiltonian is defined as shown in Figure 1-A. The $\mathrm{x}, \mathrm{y}$ and $\mathrm{z}$ axis are taken along the edges of the cubic unit cell. The quantisation axis is the $\mathrm{z}$ axis. Because of symmetry only the direction within the tetrahedron delimited by the four-fold $(<100>)$ the two-fold $(<110>)$ and the three-fold $(<111>)$ directions as shown in Figure 1-A is considered. The polar angles of the input vector $\mathrm{R}$ are called $\theta$ and $\phi$ and the computed polar angles of J are labelled $\theta^{\prime}$ and $\phi^{\prime}$ as illustrated in Figure 1-B The self-consistent condition is obtained when $\left(\theta=\theta^{\prime}\right)$ and $\left(\phi=\phi^{\prime}\right)$.

The large amount of experimental data available in the literature shows that apart from few exceptions the direction of spontaneous magnetisation at low temperature is along one of the symmetry axis. It is therefore reasonable to assume that the direction of spontaneous magnetisation is along one of the symmetry axis except in the regions of the $\{\mathrm{R}, \mathrm{x}\}$ parameter space near the boundaries between regions with different orientations of spontaneous magnetisation. The computation was performed in two steps. In the first step a diagram indicating, for each value of $(\mathrm{R}, \mathrm{x})$, the crystallographic orientation along which the magnetisation has the lowest energy was obtained. In the second step a refined investigation of the regions of the $\{R, x\}$ parameter space in the vicinity of the boundaries between the regions in the diagrams obtained in the first step was carried out. 


\subsection{The magnetic orientation diagram}

In the first step, the value of $x$ was scanned from -1 to +1 in steps of 0.01 at fixed values of $|R|$. For each value of $(x,|R|)$ the energy of the ground state corresponding to $R$ parallel to each of the three principal directions was computed. The software returned the orientation giving the lowest energy for the ground state. This orientation was taken as the direction of spontaneous magnetisation. The diagrams shown in Figure A-1 to A-11 in appendix 2 for the different values of $\mathrm{J}$ were thus obtained. The range and increment steps for $|\mathrm{R}|$ were decided on a case-by-case basis for each value of J. In all cases for the upper bound we used realistic values observed experimentally which do not exceed 80 in ferromagnetic compounds such as the $\mathrm{RFe}_{2}$ compounds [7]. In most cases we present our computations with values of $|\mathrm{R}|$ less than 30 . Above this value nothing interesting has been observed. Except in cases where the ground state in the absence of magnetic interactions is degenerate and contains a conjugate magnetic doublet in the limit very small $|\mathrm{R}|$ the value of $\langle\mathrm{J}\rangle$ goes to zero and therefore the magnetic anisotropy becomes negligible; rounding errors occurring in the computation can lead to erroneous results. Therefore, we do not report any computations with values of $|\mathrm{R}|$ less than 0.1 . All the computations were done in double precision.

\subsection{The boundaries}

In the second step we have investigated the boundaries between the regions of the $\{\mathrm{R}, \mathrm{x}\}$ parameter space with different orientations of spontaneous magnetisation. There are two fundamentally different reasons why a change of direction of spontaneous magnetisation can occur. The first one is trivial and is observed if there is an energy level cross-over. In this case there is a change of ground state at the cross-over; if the two states involved have different orientations of spontaneous magnetisation there is an abrupt change of direction of spontaneous magnetisation at the cross-over. The second and more interesting situation is where the evolution of the components of the ground state as function of $(\mathrm{R}, \mathrm{x})$ yields a different stable orientation for J. For all the boundaries shown in Figures A-1 to A-11 the evolution of the energy of the ground and first exited states across the boundary has been investigated at selected points. It has been found that there are no energy level cross-over at the transitions. The change in direction of spontaneous magnetization is in all cases due to a continuous evolution of the components of the ground state. In this case two types of behaviour can be expected at the boundaries. There could be a region where there is a continuous rotation of the direction of spontaneous magnetisation from one axis to the other or there could be a sudden jump from one orientation to the other at a critical value of $(\mathrm{R}, \mathrm{x})$. Our work has shown that both types of behaviour are observed and that there are two possible ways in which the sudden reorientation can occur. The three types of reorientation will be discussed in more details in the next section.

In order to minimize the amount of computer time required we have made the assumption that any interesting behaviour will be observed in the plane defined by the orientations on each side of the boundary under investigation. The computations were therefore restricted to those planes with only a few spot calculations outside the plane to ascertain the validity of the assumption. A systematic scans approach to the computation rather than a minimisation routine was used. For any given value of $(R, x)$ near the boundary the polar angles $\theta$ and $\phi$ of $\mathbf{R}$ were scanned in steps of 0.1 degrees in the plane defined by the two orientations of interest. At each step the energy of the ground state and the polar angles $\theta^{\prime}$ and $\phi^{\prime}$ of the direction of $\mathbf{J}$ were calculated. The data was then analysed manually to find the self-consistent orientation with the lowest energy.

\section{Results and discussion}

The orientation diagram for the direction of spontaneous magnetisation was mapped for the values of $\mathrm{J}$ between 3 and 8 in steps of $1 / 2$. The diagrams are presented in Figures A-1 to A-11 in appendix 2. The label in each region of the diagrams gives the direction of spontaneous magnetisation in that region of the $\{\mathrm{R}, \mathrm{x}\}$ parameter space. Typical behaviours at the boundaries between regions of different orientations of spontaneous 


\section{CARBONI, E. AL-ABRY and S. ARAFIN}

magnetisation are given in Figures 2 to 4 . The types of reorientation at each boundary are summarized in Table 1 .

\subsection{The orientation diagrams}

For all the values of $\mathrm{J}$ investigated, it is found that each one of the three principal crystallographic axis, $<100>$, $<110>$ and $<111>$ can be the orientation of spontaneous magnetisation for some values of the $(\mathrm{R}, \mathrm{x})$ parameters. For a dominant positive fourth-order term in the crystal field $(\mathrm{x} \sim+1)$ the spontaneous magnetisation is always along a three-fold axis $(<111>)$. For a dominant negative fourth-order term in the crystal field $(\mathrm{x} \sim-1)$ the spontaneous magnetisation is always along a four-fold axis $(<100>)$. Regions with respectively the four-fold axis and the three-fold axis as the direction of spontaneous magnetisation are never found adjacent to each other except in the limit $\mathrm{R}=0$. This observation implies that a direct reorientation between the $<111>$ and the $<100>$ cannot be observed as a function of $\mathrm{x}$ or R. For $\mathrm{J}=3$ and $\mathrm{J}=3.5$ there are only three regions in the diagram, one at the top $(\mathrm{x} \sim+1)$ and one at the bottom $(\mathrm{x} \sim-1)$ where three-fold axis and the four-fold axis are respectively the directions of spontaneous magnetisation and the region between these two where the direction of spontaneous magnetisation is the two-fold axis. This arrangement of the three main open regions is found for all values of $J$ and is observed even at unrealistic large values of $R(R>1000)$. Note that for $\mathrm{J}=3$ and $\mathrm{J}=3.5$ the boundaries converge to the same point at $\mathrm{R}=0$ therefore, in materials where the exchange interaction is weak, the two fold axis is rarely found to be the direction of spontaneous magnetisation for ions with $J=3$ or 3.5. For $J \geq 4$ there are, within the region where direction of spontaneous magnetisation is the two-fold axis some closed areas with different directions of spontaneous magnetisation. These closed areas are always bound on one side by the $\mathrm{R}=0$ axis. There are no common boundaries between two closed areas except at the limit of $\mathrm{R}=0$. Finally we note in passing that nothing particular is observed around the value of $\mathrm{R}$ $=1$ where there is a change in the relative strengths of the electrostatic and magnetic interactions.

\subsection{The boundaries}

As already mentioned three types of reorientation transitions have been observed; the continuous transition with a gradual reorientation of the direction of spontaneous magnetization and two types of discontinuous reorientations with a sudden change of the direction of spontaneous magnetization at a critical value of $(\mathrm{R}, \mathrm{x})$.

We discuss first the continuous reorientation. In this case there is near the boundary a band where a self consistent orientation of $\mathbf{R}$ is found in a direction other than the principal crystallographic directions. That intermediate self consistent orientation also corresponds to a minimum in the energy of the ground state. As an example for this type of transition the results of the computation for the point $|\mathrm{R}|=15$ and $\mathrm{x}=-0.25$ at the $<100>/<110>$ boundary for $\mathrm{J}=4.5$ are given in Figure 2. For the value of $\theta=25.4^{\circ}$ it is found that $\theta^{\prime}$ is equal to $\theta$ (Figure 2A). This value of $\theta$ also corresponds to a minimum in the energy of the ground state (Figure 2B). Therefore that intermediate orientation is the orientation of spontaneous magnetization. The direction of spontaneous magnetization thus obtained for different values of $\mathrm{x}$ varying from $\mathrm{x}=-0.24$ to $\mathrm{x}=-0.26$ at $\mathrm{R}=15$ is given in Figure 2c. There is a continuous rotation of the direction of spontaneous magnetization. The curve of the self-consistent orientation as function of the parameter $\mathrm{x}$ has infinite slope at the two critical values of $\mathrm{x}$. One could see an analogy between Figure 2c and the behavior observed in the case of a temperature driven spin reorientation however, in the present case the reorientation is not driven by temperature, it is driven by the evolution of the ground state of the ion as the relative strengths of the electrostatic and magnetic interaction is altered. This type of reorientation is called type $C$ in Table 1. 

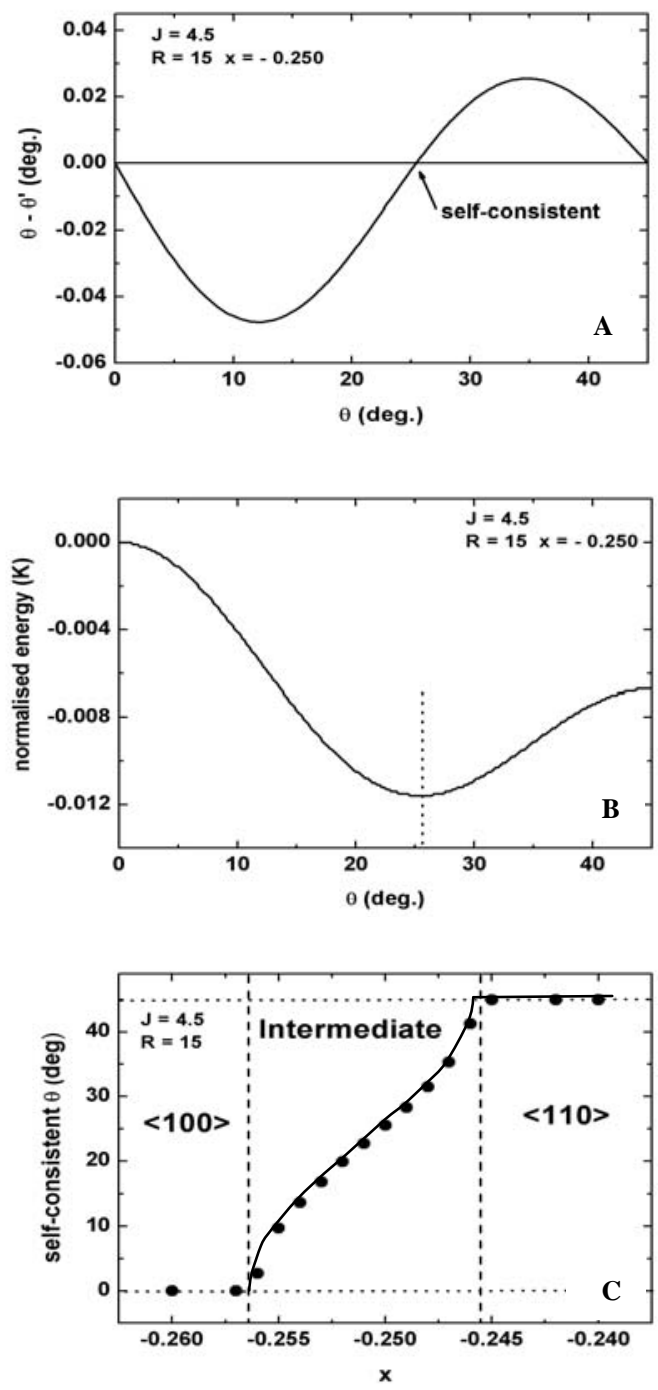

Figure 2. The type $C$ continuous reorientation for $J=4.5$ at $R=15$. A: at $x=-0.25$ the value of $\left(\theta-\theta^{\prime}\right)$ is zero when $\theta=25.4$ degrees. $\mathrm{B}$ : The minimum energy for the ground state is also found for $\theta=25.4$ degrees. $\mathrm{C}$ : The evolution of the direction of spontaneous magnetisation across the boundary. There is a well defined band from $\mathrm{x}$ $=-0.2563$ to $\mathrm{x}=-0.2456$ in which the direction of spontaneous magnetisation is at an intermediate angle.

An intermediate self consistent orientation of $\mathbf{R}$ is also found near the boundary in the first type of discontinuous reorientation however this self-consistent orientation does not correspond to a minimum in the energy of the ground state; it corresponds to a maximum. In this type of reorientation there is a band near the boundary in which there exists an intermediate unstable equilibrium orientation for the magnetization but the change-over from of the direction of spontaneous magnetization from one axis to the other occurs at a critical value of $(\mathrm{R}, \mathrm{x})$. An example of this type of transition for $\mathrm{J}=5.5$ at $\mathrm{R}=20$ and $\mathrm{x} \sim 0.1$ near the $<110>/<111>$ boundary is shown in Figure 3. For easy comparison, the energy curves presented in Figure 3B have been shifted so that so that the value at $\varphi=0$ is zero for all the curves. For $\mathrm{x}=0.110$ the $<110>$ direction $(\varphi=0)$ corresponds 


\section{CARBONI, E. AL-ABRY and S. ARAFIN}

to the lowest energy, it is the direction of spontaneous magnetization and for $\mathrm{x}=0.100$ the $<111>$ direction $(\varphi=$ 45 ) is the direction of spontaneous magnetization. There is a value of $\mathrm{x}$ between 0.100 and 0.110 for which the energy with $\mathrm{R}$ along the $\langle 110>$ direction is the same as that with $\mathrm{R}$ along the $<111>$ direction; both directions can therefore be direction of spontaneous magnetization. At that value of $\mathrm{x}$ there is a sudden change of the direction of spontaneous magnetization. This type of reorientation with a critical value of $(\mathrm{R}, \mathrm{x})$ at which the two axis are direction of spontaneous magnetization is called type Da in Table 1.

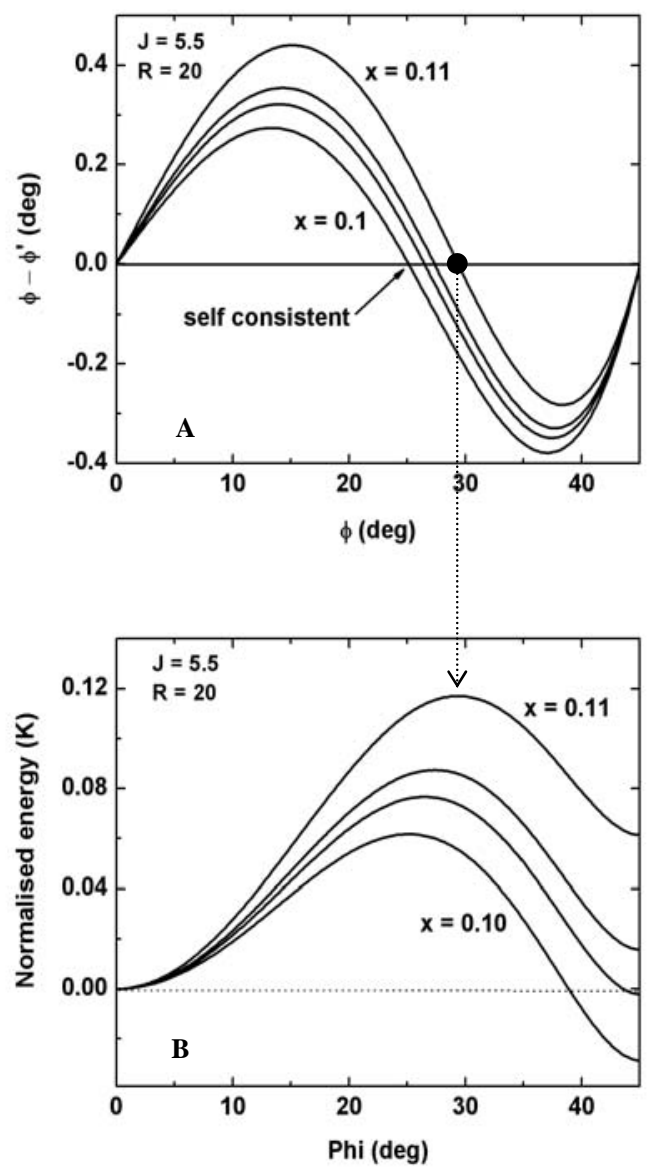

Figure 3. The type Da discontinuous reorientation for $\mathrm{J}=5.5$ at $\mathrm{R}=20$. A: there is a well defined band in which an intermediate self consistent orientation of $\mathrm{R}$ can be found however, this orientation corresponds to a maximum in the energy of the ground state. $\mathrm{B}$ : there is a value of $\mathrm{x}$ for which the magnetisation along the $<100>$ direction has the same energy as it has when along the $<111>$ direction. Both directions can be direction of spontaneous magnetization.

The third type of reorientation is illustrated in Figure 4. As in Figure $3 b$ the energy curves in Figure 4a have been shifted so that the value at $\theta=0$ is zero for all the curves. For all the values of $\mathrm{x}$ near the boundary it is found that the energy varies monotonously (Figure $4 a$ ) and that the value of $\left(\theta-\theta^{\prime}\right)$ does not change sign (Figure $4 \mathrm{~b}$ ) when the polar angle of $\mathrm{R}$ is scanned from one axis to the other. There are no intermediate self- 
consistent orientations in the plane defined by the $\langle 100\rangle$ and the $<110>$ axis. However, there is a critical value of $\mathrm{x}$ for which all the orientations in the plane have the same energy and $\theta^{\prime}$ is equal to $\theta$ for all values of $\theta$. At that critical value of $(\mathrm{R}, \mathrm{x})$ all the orientations in the plane can be direction of spontaneous magnetization. This type of reorientation is called type $D p$ in Table 1.

The type of all the boundaries in Figures A-1 to A-11 are summarised in Table 1. All the reorientations between the $\langle 110\rangle$ and the $\langle 111\rangle$ directions are of type $\mathrm{Da}$. The reorientation between the $\langle 100\rangle$ and the $<110>$ directions are continuous except for $\mathrm{J}$ less or equal to 4 where they are of type Dp.
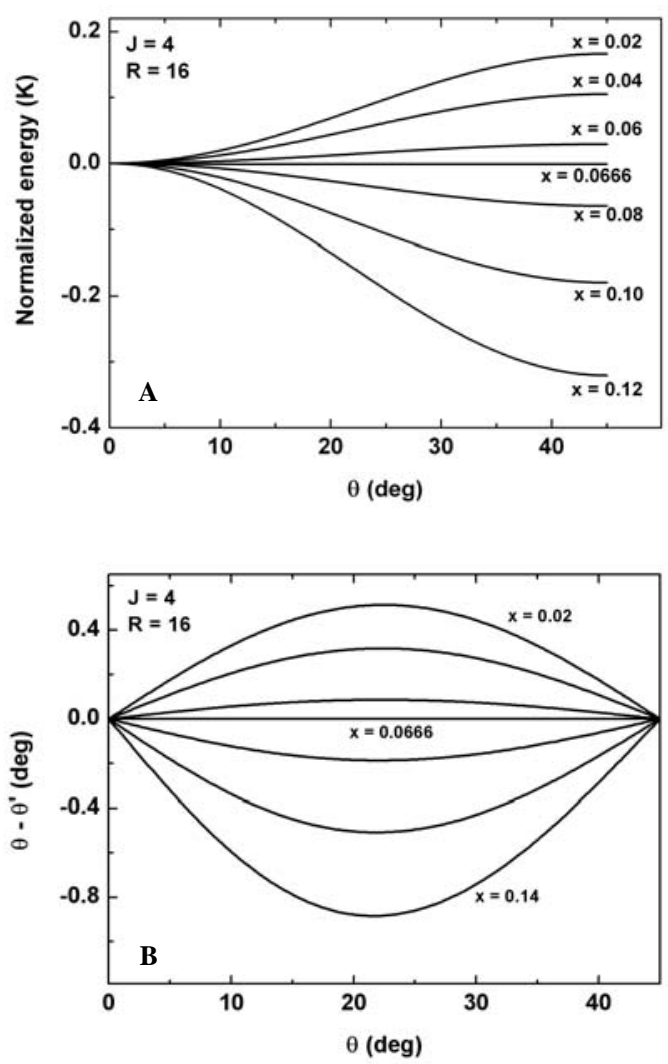

Figure 4. The type $\mathrm{Dp}$ of discontinuous reorientation for $\mathrm{J}=4$ at $\mathrm{R}=16$. A: there is no self consistent orientation of R. B: at a critical value of $\mathrm{x}$ all the directions in the plane defined by the $<100\rangle$ and the $\langle 110\rangle$ axis can be direction of spontaneous magnetisation.

The width $\Delta \mathrm{x}$ at constant $\mathrm{R}$ of the reorientation band in the type $\mathrm{C}$ boundary can vary from 0.04 where it is easily identified to about $10^{-6}$ where it may be mistakenly seen as a type Dp. For example, the width $\Delta \mathrm{x}$ of the boundary of the closed domain in the diagram for $\mathrm{J}=7$ is $5 \times 10^{-6}$ at $\mathrm{R}=0.8$. The energy difference between the three self consistent orientations in the plane is $1 \times 10^{-6}$ that is almost the limit of confidence of our computations. An illustration of the limits of our computation for the type Dp boundaries is given in Table 2 for $J=3$. For both values of $x$ given in the Table the energy of the ground state varies monotonously as the angle $\theta$ is varied from zero to forty five degrees; the energy difference between the magnetisation being along the $<100>$ and being 


\section{CARBONI, E. AL-ABRY and S. ARAFIN}

along the $\langle 110\rangle$ axis is about $5 \times 10^{-6}$. A change of a change of $5 \times 10^{-10}$ in the value of $\mathrm{x}$ causes the change of the direction of spontaneous magnetisation from one axis to the other.

Table 1. The types of reorientation. In the type $\mathrm{C}$ there is a continuous rotation of the direction of spontaneous magnetization from one axis to the other. In the type Da there is a critical value of the parameters where both axes can be direction of spontaneous magnetization. In the type Dp there is a critical value of the parameters where all the directions in the plane defined by the two axis involved are directions of spontaneous magnetization.

\begin{tabular}{|c|c|c|c|c|c|c|}
\hline J & Boundary & Type & & J & Boundary & Type \\
\hline 3 & $<100>/<110>$ & $\mathrm{Dp}$ & & 6 & $<100>/<110>$ & $\mathrm{C}$ \\
\hline & $<110>/<111>$ & $\mathrm{Da}$ & & & $<110>/<111>$ closed & $\mathrm{Da}$ \\
\hline 3.5 & $<100>/<110>$ & $\mathrm{Dp}$ & & & $<110>/<100>$ closed & $\mathrm{C}$ \\
\hline & $<110>/<111>$ & $\mathrm{Da}$ & & & $<110>/<111>$ & $\mathrm{Da}$ \\
\hline 4 & $<100>/<110>$ & $\mathrm{Dp}$ & & 6.5 & $<100>/<110>$ & $\mathrm{C}$ \\
\hline & $<110>/<111>$ closed & $\mathrm{Da}$ & & & $<110>/<111>$ closed & $\mathrm{Da}$ \\
\hline & $<110>/<111>$ & $\mathrm{Da}$ & & & $<110>/<100>$ closed & $\mathrm{C}$ \\
\hline 4.5 & $<100>/<110>$ & $\mathrm{C}$ & & & $<110>/<111>$ & $\mathrm{Da}$ \\
\hline & $<110>/<111>$ closed & $\mathrm{Da}$ & & 7 & $<100>/<110>$ & $\mathrm{C}$ \\
\hline & $<110>/<100>$ closed & $\mathrm{C}$ & & & $<110>/<111>$ closed & $\mathrm{Da}$ \\
\hline & $<110>/<111>$ & $\mathrm{Da}$ & & & $<110>/<100>$ closed & $\mathrm{C}$ \\
\hline 5 & $<100>/<110>$ & $\mathrm{C}$ & & & $<110>/<111>$ & $\mathrm{Da}$ \\
\hline & $<110>/<111>$ closed & $\mathrm{Da}$ & & 7.5 & $<100>/<110>$ & $\mathrm{C}$ \\
\hline & $<110>/<100>$ closed & $\mathrm{C}$ & & & $<110>/<111>$ closed & $\mathrm{Da}$ \\
\hline & $<110>/<111>$ & $\mathrm{Da}$ & & & $<110>/<100>$ closed & $\mathrm{C}$ \\
\hline 5.5 & $<100>/<110>$ & $\mathrm{C}$ & & & $<110>/<111>$ & $\mathrm{Da}$ \\
\hline & $<110>/<111>$ closed & $\mathrm{Da}$ & & 8 & $<100>/<110>$ & $\mathrm{C}$ \\
\hline & $<110>/<100>$ closed & $\mathrm{C}$ & & & $<110>/<111>$ closed & $\mathrm{Da}$ \\
\hline & $<110>/<111>$ & $\mathrm{Da}$ & & & $<110>/<100>$ closed & $\mathrm{C}$ \\
\hline & & & & & $<110>/<111>$ & $\mathrm{Da}$ \\
\hline
\end{tabular}

Table 2. The limit of the computation for the type Dp boundary. A change of a change of $5 \times 10^{-10}$ causes the change of the direction of spontaneous magnetisation from one axis to the other.

\begin{tabular}{|c|c|c|}
\hline $\mathbf{X}$ & $\mathbf{E}(<\mathbf{1 0 0}>)$ & $\mathbf{E}(<\mathbf{1 1 0}>)$ \\
\hline-0.1694136605 & -67.565397765 & -67.565397771 \\
\hline-0.1694136610 & -67.565397768 & -67.565397762 \\
\hline
\end{tabular}

Should any of the boundaries labelled $\mathrm{Dp}$ in Table 1 be continuous, the reorientations would occur over a range of values of $\mathrm{x}$ smaller than $5 \times 10^{-10}$.

Finally we note that a situation where there is a minimum in the energy that does not correspond to a self consistent orientation has not been observed.

\section{Conclusion}

Since the parameters $\mathrm{R}$ and $\mathrm{x}$ are not easily accessible to the experimentalist one might ask if the work presented can have any use for the interpretation of experimental data. The parameter $\mathrm{R}$ can in principle be 
varied by the application of an external magnetic field; however, the applied field must be along the selfconsistent orientation otherwise it will introduce anisotropy and invalidate the tenet of our analysis. However, many experimentalists make use of the fact that because the lanthanide ions have identical outer electrons-shells the ion under investigation can be placed in a homologue compound with a different rare earth. In particular the magnetic interactions can be reduced by substituting a magnetic ion by yttrium, lanthanum or lutetium (although yttrium is not strictly part of the lanthanide series) or increased by introducing gadolinium. It is usually assumed that yttrium, lanthanum and lutetium have no magnetic moment and that the substitution does not affect significantly the crystal field at the site of the ion of interest; it is also assumed that gadolinium does not interact with the crystal field. These assumptions have been shown not to be tenable in many compounds, too numerous to be reviewed here; however, dilution and substitution has enable researchers to clarify so many problems that the assumptions can be justified as a first approximation.

An interesting compound is $\mathrm{HoCo}_{2}$. The $\mathrm{Ho}^{3+}(\mathrm{J}=8)$ ion is at a site of cubic symmetry. According to Gignoux et al. (1975) the direction of spontaneous magnetisation at low temperature is the $<110>$ direction; the parameters $\mathrm{R}=45.8$ and $\mathrm{x}=-0.4665$ derived from the data of Gignoux et al. (1975) place the compound in the area corresponding to the $\langle 110\rangle$ orientation in our diagram close to the boundary with a continuous reorientation. A small increase in $\mathrm{R}$ will causes the compound to magnetise along the $<100>$ axis. How ever, the parameters given by Aubert et al. (1978) place the compound just inside the reorientation area and those published by Castet et al. (1982) place the compound on the other $(<100>)$ side of the boundary.

The author and co-workers at the University of Manchester (UK) [11 and 12] have investigated by ${ }^{165} \mathrm{Ho}$ nmr the series of compounds $\mathrm{Y}_{\mathrm{x}} \mathrm{Ho}_{1-\mathrm{x}} \mathrm{Co}_{2}, \mathrm{Gd}_{\mathrm{x}} \mathrm{Ho}_{1-\mathrm{x}} \mathrm{Co}_{2}$ and $\mathrm{Gd}_{\mathrm{x}} \mathrm{Y}_{\mathrm{y}} \mathrm{Ho}_{1-\mathrm{x}-\mathrm{y}} \mathrm{Co}_{2}$ at $1.3 \mathrm{~K}$ in zero applied field. The direction of spontaneous magnetisation can be inferred from the behaviour of the hyperfine parameters [13 and 14]. It was found that $\mathrm{HoCo}_{2}$ is just inside the reorientation region, it magnetises at a small angle to the $<110>$ axis. $\mathrm{Y}_{\mathrm{x}} \mathrm{Ho}_{1-\mathrm{X}} \mathrm{Co}_{2}$ for $\mathrm{x}=0.05$ (smaller $\mathrm{R}$ ) magnetises along the $<110>$ direction. The compounds $\mathrm{Gd}_{\mathrm{x}} \mathrm{Ho}_{1-\mathrm{x}} \mathrm{Co}_{2}$ for $\mathrm{x}<0.40$ are inside the reorientation region, the angle between the direction of spontaneous magnetisation and the $\langle 100\rangle$ axis decreases with increasing concentration of gadolinium (increasing R). For $\mathrm{x}>=0.40$ the spontaneous magnetisation is along the $<100>$ direction. Finally the compound $\mathrm{Gd}_{\mathrm{x}} \mathrm{Y}_{\mathrm{y}} \mathrm{Ho}_{1-\mathrm{x}-\mathrm{y}} \mathrm{Co}_{2}$ with an average value of $\mathrm{R}$ corresponding to the reorientation region was observed to magnetise at a small angle to the $<100>$ direction. Details of this experiment and the analysis will be published elsewhere.

The knowledge of the position of the compound $\mathrm{HoCo}_{2}$ on the diagram is therefore of some help in ascertaining or controlling the direction of spontaneous magnetisation in this series of compounds.

\section{Acknowledgements}

The author C C is indebted to Dr M A H McCausland D, St P Bunbury and O. Prakash for discussions during his time at the University of Manchester.

E Al-Abry is grateful to the Ministry of Higher Education of the Sultanate of Oman for providing a scholarship.

Appendix 1: Stevens operators equivalent and the coefficients $F_{4}$ and $F_{6}$

The Stevens equivalent operators for the non vanishing terms in the crystal field Hamiltonian for a site of cubic symmetry.

$$
\begin{aligned}
O_{4}^{0}= & 35 J_{z}^{4}-[30 J(J+1)-25] J_{z}^{2}-6 J(J+1)+3 J^{2}(J+1)^{2} \\
O_{4}^{4}= & \frac{1}{2}\left(J_{+}^{4}+J_{-}^{4}\right) \\
O_{6}^{0}= & 231 J_{z}^{6}-105[3 J(J+1)-7] J_{z}^{4}+\left[105 J_{z}^{2}(J+1)^{2}-525 J(J+1)+294\right] J_{z}^{2} \\
& \quad-5 J^{2}(J+1)+40 J^{2}(J+1)^{2}-60(J(J+1) \\
O_{6}^{4}= & \frac{1}{4}\left[11 J_{z}^{2}-J(J+1)-38\right]\left(J_{+}^{4}+J_{-}^{4}\right)+\frac{1}{4}\left(J_{+}^{4}+J_{-}^{4}\right)\left[11 J_{z}^{4}-J(J+1)-38\right]
\end{aligned}
$$




\section{CARBONI, E. AL-ABRY and S. ARAFIN}

The value of the coefficients $\mathrm{F}_{4}$ and $\mathrm{F}_{6}$ given by Lea. Leask and Wolf.

\begin{tabular}{|c|c|c|}
\hline $\mathbf{J}$ & $\mathbf{F}_{\mathbf{4}}$ & $\mathbf{F}_{\mathbf{6}}$ \\
\hline 3 & 15 & 180 \\
\hline 3.5 & 60 & 1260 \\
\hline 4 & 60 & 1260 \\
\hline 4.5 & 60 & 2520 \\
\hline 5 & 14 & 1260 \\
\hline 5.5 & 60 & 3780 \\
\hline
\end{tabular}

\begin{tabular}{|c|c|c|}
\hline $\mathbf{J}$ & $\mathbf{F}_{\mathbf{4}}$ & $\mathbf{F}_{\mathbf{6}}$ \\
\hline 6 & 60 & 7560 \\
\hline 6.5 & 60 & 7560 \\
\hline 7 & 60 & 3780 \\
\hline 7.5 & 60 & 13860 \\
\hline 8 & 60 & 13860 \\
\hline
\end{tabular}

Table A 1 . The values of the coefficients $\mathrm{F}_{4}$ and $\mathrm{F}_{6}$ corresponding to different values of $\mathrm{J}$.

Appendix 2: The orientation diagrams
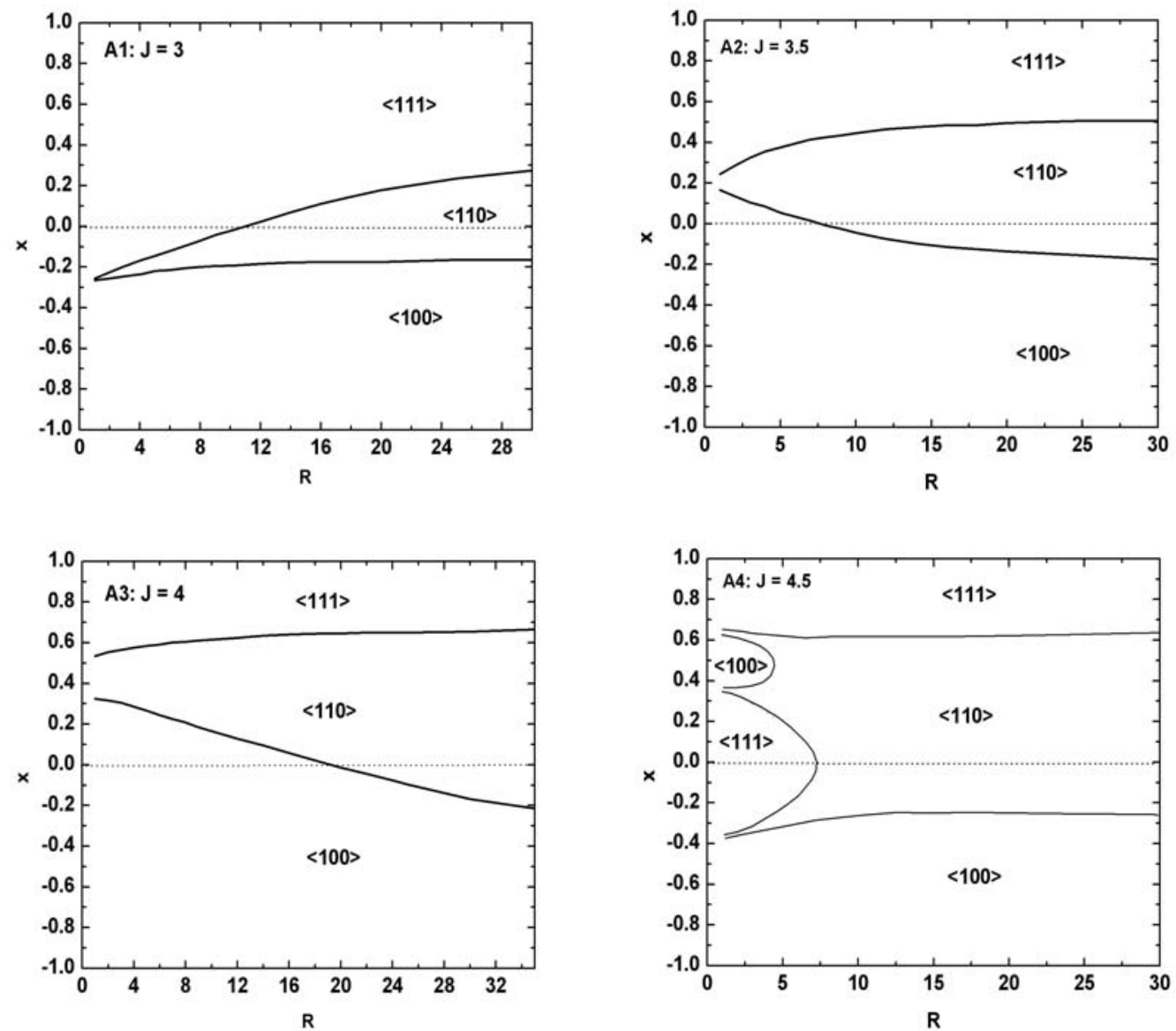
THE DIRECTION OF SPONTANEOUS MAGNETISATION OF LANTHANIDE IONS
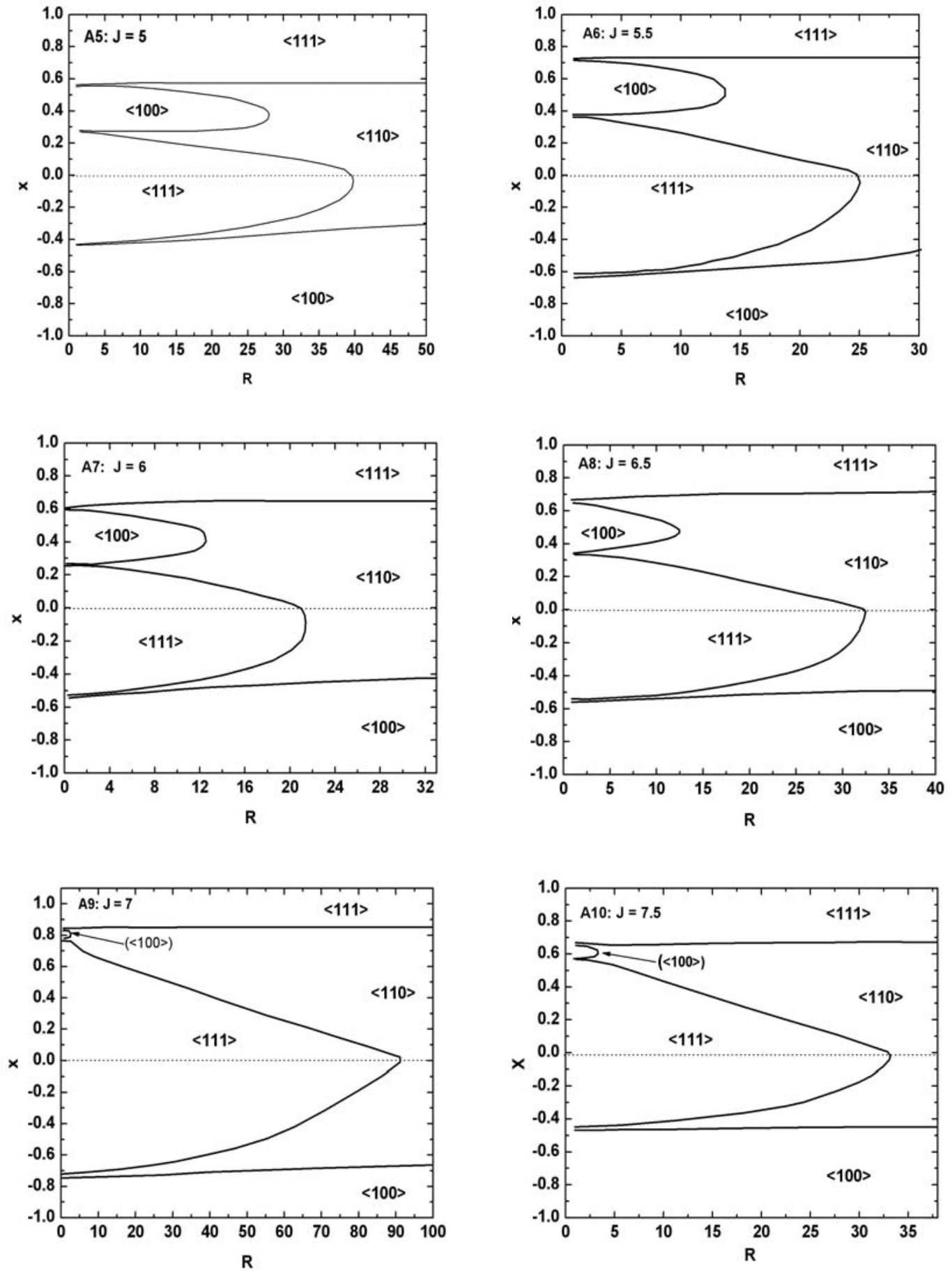


\section{References}

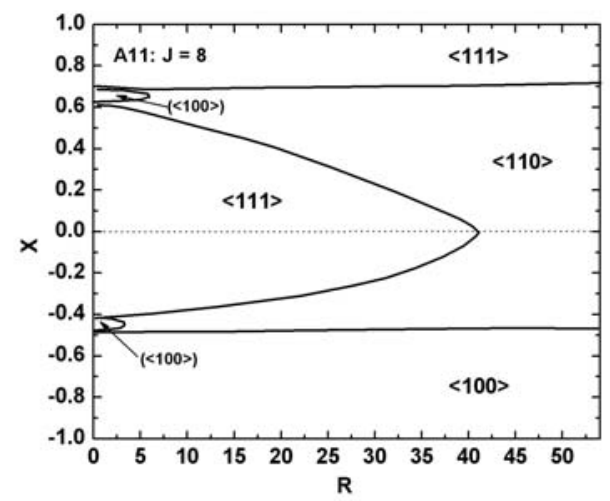

AUBERT, G., GIGNOUX, D., GIVORD, F., LEMAIRE, R. and MICHELUTTI, B. 1978. Magnetization Reorientation in $\mathrm{HoCo}_{2}$. Solid State Com., 25: 85.

BUNBURY, D.ST.P., CARBONI, C., McCAUSLAND, M.A.H., CONE, R., MROCZKOWSKY, S. and ROSS, J.W. 1995. The hyperfine splitting of the ground state and first excited state of holmium in yttrium hydroxide. J Phys. Condens. Matte, 7, 6125-36.

BUNBURY, D.ST.P., CARBONI, C., MCCAUSLAND, M.A.H. and PRAKASH, O.1986. Spin reorientation in $\mathrm{HoCo}_{2}$, an NMR study, Proc. XXIII Congress Ampere on Magnetic Resonance (Istituto Superiore di Sanita, Roma) p. 296.

CARBONI, C. 1984. Diploma Report, Design of a microwave resonator to measure the NMR of ${ }^{3+} \mathrm{Ho}$ in metallic single crystals. University of Manchester, unpublished.

CARBONI, C., GIGNOUX, D. and ROSS, J.W. 1996. The field dependence of the hyperfine splitting of terbium in $\mathrm{TbNi}_{5}$, J. Phys Condens. Matter, 8: 1763-74.

CARBONI, C., McCAUSLAND, M.A.H., BUNBURY, D.S.P., REID, B.L., LATAIFEH, M. and ABELL, J.S. 1992. Crystal-field anisotropy of $\mathrm{Tb}^{3+}, \mathrm{Ho}^{3+}$, and $\mathrm{Tm}^{3+}$ in gadolinium. J. Magn. Magn. Matter. 104107: 1513.

CASTET, A., GIGNOUX, D. and HENNION, B. 1982. New aspects of the magnetic excitations in $\mathrm{HoCo}_{2}$. Phys. Rev. B25: 337.

GERMANO, D.J. and BUTERA, R.A. 1981. Heat capacity of, and crystal-field effects in, the $\mathrm{RFe}_{2}$ intermetallic compounds $(\mathrm{R}=\mathrm{Gd}, \mathrm{Tb}$, Dy, Ho, Er, Tm, and Lu) Phys. Rev. B, 24(1): 3912.

GIGNOUX, D., GIVORD, F., LEMAIRE, R. 1975. Magnetic properties of single crystals of $\mathrm{GdCo}_{2}, \mathrm{HoNi}_{2}$, and $\mathrm{HoCo}_{2}$; Phys. Rev. B12: 3878.

LEA, K.R., LEASK, J.M. and WOLF, W.P. 1962. The raising of angular momentum degeneracy of f-electrons by cubic crystal field, Journal of the Physics and Chemistry of Solids, 23: 1381-1405.

McCAUSLAND, M.A.H, and MAKENZIE, I.S. 1980. Nuclear Magnetic Resonance in Rare earth Metals, Taylor and Francis Ltd., London.

MORRISSON, C.A. and LEAVITT, R.P. 1982. Handbook of Physics and Chemistry of Rare Earths, Vol 5, Gschneider, K.A and Eyring, L eds. North Holland Publishing Company.

PRAKASH, O., BUNBURY, D.ST.P., McCAUSLAND, M.A.H. 1984. On the ground state of $\mathrm{Ho}^{3+}$ in ferromagnetic $\mathrm{HoAl}_{2}, Z$ Phys B58, 39.

STEVENS, K.W.H. 1952. Matrix elements and operator equivalents connected with the magnetic properties of rare-earth ions, Proceedings of the Physical Scociety, A, 65: 209-215.

Received 11 October 2006

Accepted 29 January 2007 